\title{
Lengthening and shortening processes in Korean
}

\author{
Hyunsook Kang ${ }^{1}$ Tae-kyung Kim ${ }^{2, *}$ \\ ${ }^{I}$ Department of English Language and Culture, Hanyang University, Ansan, Korea \\ ${ }^{2}$ Center for Integrated General Education, Hanyang University, Ansan, Korea
}

\begin{abstract}
This study examines the duration of Korean lax and tense stops in the prosodic word-medial position, their interactions with nearby segments, and the phonological implications of these interactions. It first examines the lengthening of consonants at the function of the short lax stop. Experiment 1 shows that the sonorant $\mathrm{C} 1$ is significantly longer before a short lax stop $\mathrm{C} 2$ than before a long tense stop. Experiment 2 shows that the short lax stop C1 cancels the contrast between the lax and tense obstruent at C2, making them appear as long tense obstruents (Post-Stop Tensing Rule). We suggest that such lengthening phenomena occur in Korean to robustly preserve the contrastive length difference between C and CC. Second, this study examines the vowel shortening, known as Closed-Syllable Vowel Shortening, before a long tense stop or before the consonant sequence. Experiment 3 suggests that it be interpreted as temporal adjustment to make the interval from the onset of a vowel to the onset of the following vowel of near-equal length. Conclusively, we suggest that Korean speech be planned and controlled with two specific intervals. One is the duration of contrastive consonant intervals between vowels, and the other is the duration from the onset of a vowel to the onset of the following vowel.
\end{abstract}

Keywords: Korean lax and tense stops, lengthening of consonants, Post-Stop Tensing Rule, vowel shortening

\section{Introduction}

Korean has three types of stops: a lax, a tense, and an aspirated stop. In the AP-initial position, they are distinguished by voice-onset time and the pitch of the following vowel (Jun, 1993; Kim, 2000, etc.).

In the AP-medial position, these acoustic cues are also active but not as effective as they are in the word-initial position (cf. Jun, 1993, 1995). Instead, some other acoustic cue like closure duration of the stops plays an important role, especially in differentiating between a tense stop and a lax stop. A tense stop shows the longest closure duration, the closure duration of which is more than twice longer than that of a lax stop (cf. Silva, 1992; Han, 1996; etc.).

Many perceptual studies have shown the importance of closure duration in distinguishing the intervocalic tense stop from the lax stop. Han (1996) and many others have shown that a tense and a lax stop in the intervocalic position can be confused for each other if the closure duration varies. Thus, in order to keep a lax and a tense stop apart in the intervocalic position, it is important for each stop to maintain its contrastive length of closure duration.

A phonologically long consonant is often observed to influence the duration of nearby segments, particularly the preceding vowel. Two rather different relationships are observed. Maddieson (1985)

\footnotetext{
* kimtk@hanyang.ac.kr, Corresponding author

Received 29 July 2020; Revised 16 September 2020; Accepted 16 September 2020

(c) Copyright 2020 Korean Society of Speech Sciences. This is an Open-Access article distributed under the terms of the Creative Commons Attribution NonCommercial License (http://creativecommons.org/licenses/by-nc/4.0) which permits unrestricted non-commercial use, distribution, and reproduction in any medium, provided the original work is properly cited.
} 
observes that some languages with a contrast between single and geminate consonants exhibit an inverse relationship between the length of consonant and the length of the preceding vowel. For instance, in languages like Kannada, short vowels occur before long consonants (i.e., geminates) while long vowels occur before short consonants. In contrast, Japanese shows the direct relationship between the length of consonant and the length of the preceding vowel: The vowel is phonetically longer before a geminate than before a singleton (Han, 1992; Ham, 2001; Idemaru \& Guion, 2008, among others).

These two different results show that the length of consonants may influence the length of the nearby vowels possibly to control the length of the target unit. However, the exact target unit may differ depending on languages.

In addition, in some languages a phonetically long consonant may also influence the duration of the nearby segments. For instance, Lisker (1957) showed that in English, a relatively long vowel occurs before a short voiced consonant while a relatively short vowel occurs before a relatively long voiceless consonant.

Researchers have attempted to account for the durational variability of a segment in terms of languages' different rhythms such as "stress" or "syllable". It was argued that in stress-timed languages, segments undergo lengthening or shortening to make intervals between "stresses" to be near-equal, whereas in syllable-timed languages, segments undergo these processes to make successive "syllables" to be of near-equal length (Pike, 1945; Abercrombie, 1967; Oh, 2015, among others).

This line of claim, however, was not supported by clear-cut evidence. In fact, many studies have failed to find solid evidence that near-equal intervals are maintained between stresses (Bolinger, 1965 , etc.) in stress-timed languages or between successive syllables in syllable-timed languages (Delattre, 1966; etc.).

Given that there is no solid evidence for near-equal intervals between "stresses" or between "syllables", some linguists started to consider other possible units to account for the variations of segments in languages. Ramus et al. (1999) noted that speech is perceived by newborns as a sequence of vowels interspersed with noise, namely, consonants. That is, rhythms are perceived as the variation between vowels and consonants. Using a corpus of three different categories of rhythmic languages such as languages of the stress-timed category, the syllable-timed category, and the moratimed category, Ramus et al. (1999) showed that in speech vowels from languages of the same rhythmic category showed a similar amount of time as compared to vowels from languages of different rhythmic classes. Furthermore, they argued that the standard deviation of the duration of consonantal intervals is also an important factor in accounting for the durational differences of segments in three different language categories. Low et al. (2000) argue that the pairwise variability indices in vowels and the consonant intervals between vowels are other important factors in accounting different types of languages.

All these studies show that the variation of the segment duration is often accompanied by some acoustic correlates like the duration of a neighboring segment, and that durations of segments vary possibly for some rhythmic purposes like making certain salient acoustic units of near-equal length. Korean is also known for segment compression, namely that the vowel is shortened before a consonant sequence or a geminate but not before a single consonant (Oh \& Johnson, 1997; Choi \& Jun, 1998, among others). These studies have mainly focused on the effects the duration of a consonant has on the preceding vowel, but have not examined a wide range of effects in a unified way. This paper aims to investigate various phonetic or phonological effects that contrastive durations of Korean lax and tense stops may induce on nearby segments.

The phonological contexts where a Korean lax or tense stop may occur in the surface forms are quite limited in the PW-medial position. In Korean, a lax or a tense stop occurs as the first or the last segment of the morpheme and Korean syllable structure allows one onset and one coda. Therefore, a lax and a tense stop can occur either as an onset or as a coda. However, they do not phonologically contrast at the coda position since obstruents at the coda position are neutralized to lax stops (cf. $/ \mathrm{paK}^{*} / \rightarrow$ [pak] "outside"). In addition, the obstruents do not even surface at the coda position if the following segment is a sonorant due to the sonorant assimilation (cf. /kyək-li/ $\rightarrow$ [kyəy-ni] "separation", /mak-ne/ $\rightarrow$ [man-ne] "the baby of the family"). Therefore, there are only three phonological contexts in which a lax or a tense stop may surface in PW-medial position.

One such context is when a sonorant such as a lateral or a nasal occurs at $\mathrm{C} 1$ and a lax or a tense stop occurs at $\mathrm{C} 2$ either by a phonological process or by a morphological concatenation. When these consonant clusters occur in the intervocalic position, the lax stop at C2 may surface as a lax stop (cf. /ka-n-ta/ $\rightarrow$ [kanda] "go-present tense-sentence closing ending", /kal-ta/ $\rightarrow$ [kalda] "change, grind-sentence closing ending"), or as a tense stop (cf. /an-ta/ $\rightarrow$ [anT"a] "hug-sentence closing ending", /mal-tan/ $\rightarrow$ [malT"an] "terminal, the end"), depending on the morphological context.

Another context is when an obstruent occurs at $\mathrm{C} 1$, and a lax or a tense stop occurs at $\mathrm{C} 2$ either by a phonological process or by a morphological concatenation. As we noted, an obstruent at $\mathrm{Cl}$ is neutralized as a short lax stop. Therefore, a morpheme ending with an obstruent will be realized as a morpheme with a lax stop coda (cf. /os/ $\rightarrow$ [ot] "clothes", $/ \mathrm{p}^{\mathrm{h}} \mathrm{at}^{\mathrm{h}} / \rightarrow$ [ph at] "red bean"). To these morphemes, another morpheme beginning with a lax or a tense obstruent may be added as in [ot]\#[pan] "dress room", and $\left[\mathrm{p}^{\mathrm{h}}\right.$ at]\#[P*an] "red bean bread". When this happens, another neutralization takes place, neutralizing the lax and the tense obstruent at $\mathrm{C} 2$ to a long tense obstruent ${ }^{1}$ (cf. [ot]\#[pay] $\rightarrow$ [otP $^{*}$ an], and $\left[\mathrm{p}^{\mathrm{h}}\right.$ at $] \#\left[\mathrm{P}^{*} a \eta\right] \rightarrow\left[\mathrm{p}^{\mathrm{h}}\right.$ atP $\mathrm{P}^{*}$ an]). This process has been referred to as Post-Stop Tensing Rule in Korean, as the lax obstruent becomes tense after the coda stop.

The last context is when the lax or tense stop occurs in the intervocalic position (cf. /aka/ $\rightarrow$ [aga] 'baby', /aK*a/ $\rightarrow$ [aK'a] 'sometime before').

This paper consists of as follows. Section 2 will examine the acoustic effects a lax or a tense stop may have in each of the three phonological contexts mentioned above. Specifically, Experiment 1 will examine the consonant sequences composed of the sonorant

1 In this paper, the word "long" is used before a tense obstruent or stop simply to emphasize that it is more than twice longer than the corresponding lax segment. 
coda $\mathrm{C} 1$, and a lax or a tense stop at $\mathrm{C} 2$. Experiment 2 will examine the consonant sequences composed of the obstruent at $\mathrm{C} 1$, and the lax or a tense stop at $\mathrm{C} 2$. This context is well known among Korean linguists as the context of the Post-Stop Tensing Rule. Experiment 3 will examine the vowel shortening before a tense stop, argued as a case of Closed-Syllable Vowel Shortening (Choi \& Jun, 1998). Section 3 summarizes the findings in this paper and discusses the phonological implications of the findings.

\section{Experiment}

\subsection{Methodology}

\subsubsection{Participants}

Seven Seoul dialect native speakers, two male and five female speakers in their twenties, participated in this experiment. The speakers were all born and have spent the most of their lives around the Seoul area. They are currently university students. No speaker reported any speech peculiarities in speech or hearing.

\subsubsection{Stimuli}

In order to examine the temporal effects on the neighboring segments by the lax and tense stops, the list of sentences in this study included phonologically possible, lexical and non-lexical words that contained a lax or a tense stop. The test words consist of two syllables, beginning with vowel [a] and ending with vowel [a]. Between two vowels, various consonants or consonant sequences occur: a lax stop $\mathrm{VtV}$, a tense stop $\mathrm{VT} * \mathrm{~V}$, a sequence of a lax and a lax stop VttV, a sequence of a lateral and a lax stop VLtV, a sequence of a lateral and a tense stop VLT*V, a sequence of a nasal and a lax stop $\mathrm{VNtV}$, and a sequence of a nasal and a tense stop $\mathrm{VNT} * \mathrm{~V}$. The lax and the tense stop at the onset position in these stimuli show all three places of articulation. The obstruent or the nasal stop at the coda position (represented as underlined) as in $\mathrm{VttV}, \mathrm{VNtV}$, and $\mathrm{VNT} * \mathrm{~V}$ have the homorganic place of articulation with the following stop. The two-syllable test word is followed by a postpositional particle /-imnita/ as in /ataimnita/. The first vowel, and the following consonant(s) may be referred to as $\mathrm{V}, \mathrm{C} 1$, and $\mathrm{C} 2$, respectively.

The test sentences were written in Korean. Since the relationship between orthography and pronunciation in Korean is very transparent, participants had no problem pronouncing nonce words. The list of sentences is given in the appendix.

\subsubsection{Procedure}

Three speakers (two male speakers and one female speaker) were recorded individually in a sound-treated booth at Hanyang University, Seoul, by using a Shure KSM10 microphone and a Tascam (HD-P2) recorder. Four other female speakers were recorded individually in a sound-treated booth at Hanyang University, Erica, by using a Shure KSM10 microphone and a Tascam (HD-P2) recorder. Before the recording, the speakers practiced the sentences at a speed most comfortable for them. After that, each speaker made three recordings. Two tokens of each word by the same speaker were used in the analysis.

\subsubsection{Measurements}

Acoustic measurements were conducted by both authors for the durations of $[\mathrm{a}](\mathrm{V})$, a consonant at $\mathrm{C} 1$, and the consonant at $\mathrm{C} 2$. The measurements were carried out using both auditory and visual cues from the waveforms and spectrographic displays on Praat software. The glottal stop before the beginning of the first vowel [a] is excluded in the measurements of the vowel duration. The duration of $\mathrm{C} 1 \mathrm{C} 2$ was defined as the interval in between the offset of the first vowel and the onset of the second vowel. When $\mathrm{C} 1$ and $\mathrm{C} 2$ are obstruent stops, there is no clear burst between them as Korean coda stop is not released. Therefore, no attempt was made to measure $\mathrm{Cl}$ or $\mathrm{C} 2$ duration, separately. Figure 1 shows samples of measurements for /ampaimnita/ and /amP*aimnita/ by speaker HJS.

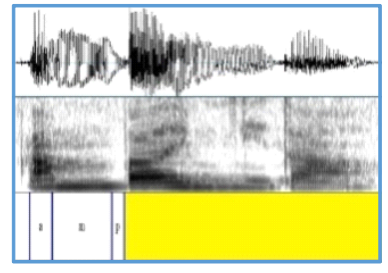

a. /ampaimnita/

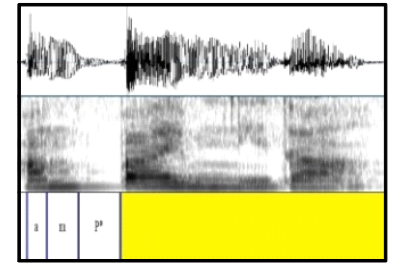

b. /amP*aimnita/
Figure 1. Spectrograms of $\mathrm{VC} 1 \mathrm{C} 2$ with relevant acoustic values

\subsection{Experiment 1}

This section examines the duration of the sonorant at $\mathrm{C} 1$ and those of the lax and the tense consonant at $\mathrm{C} 2$ in order to identify the temporal patterns of $\mathrm{C} 1$ and $\mathrm{C} 2$.

\subsubsection{Analysis}

In order to test whether $\mathrm{C} 2$ has different length depending on its type (lax or tense), and whether the duration of sonorant $\mathrm{C} 1$ is affected by the type of $\mathrm{C} 2$ (lax or tense), two separate t-tests were conducted. There were 168 tokens for each test $(7$ speakers $\times 2$ types of $\mathrm{C} 1$ (nasal or lateral) $\times 2$ types of $\mathrm{C} 2$ (lax or tense) $\times 3$ places of articulation of $\mathrm{C} 2$ (bilabial, coronal, velar) $=168$ tokens).

\subsubsection{Results}

Table 1 and Figure 2 show the results.

Table 1. Mean durations (ms) and standard deviations of $\mathrm{C} 1$ and $\mathrm{C} 2$

\begin{tabular}{c|c|c|c|c}
\hline \multirow{2}{*}{} & \multicolumn{2}{|c|}{ C1 - Lax C2 } & \multicolumn{2}{c}{ C1 - Tense C2 } \\
\cline { 2 - 5 } & Mean (ms) & $S D$ & Mean (ms) & $S D$ \\
\hline C1 (son) & 112.60 & 30.00 & 80.24 & 24.44 \\
\hline C2 (stop) & 38.60 & 15.44 & 99.01 & 24.07 \\
\hline
\end{tabular}

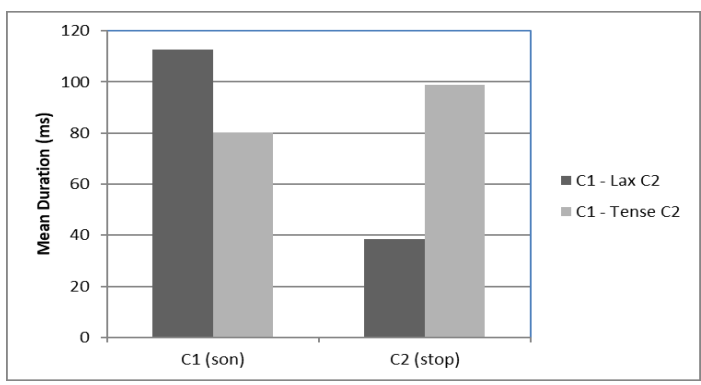

Figure 2. Mean durations of $\mathrm{C} 1$ and $\mathrm{C} 2$

The statistical results indicate that there is a significant increase in the duration of sonorant $\mathrm{C} 1$ before a lax stop $(M=112.60 \mathrm{~ms})$ 
compared to the duration of sonorant $\mathrm{C} 1$ before the tense stop $(M=80.24 \mathrm{~ms}), t(83)=13.94, p<.001$. The results also show that the tense stop C2 ( $M=99.01 \mathrm{~ms})$ shows a significantly longer closure duration than a lax stop $\mathrm{C} 2(M=38.60 \mathrm{~ms})$ does, $t(83)=29.00$, $p<.001$. In short, the sonorant $\mathrm{C} 1$ before a lax stop is significantly longer than that before a tense stop, and an onset tense stop $\mathrm{C} 2$ in a consonant sequence is more than twice as long as an onset lax stop $\mathrm{C} 1$ is.

\subsubsection{Discussion}

The statistical results for the duration of the stop at $\mathrm{C} 2$ have reconfirmed the previous finding that tense stops are twice as long as the corresponding lax stops.

Interestingly, the experimental results in this section have also shown that the duration of the sonorant at $\mathrm{Cl}$ is significantly influenced by the duration of the following stop at $\mathrm{C} 2$. Table 1 shows that the sonorants before a short lax stop $(M=112.60 \mathrm{~ms})$ were significantly longer than the corresponding sonorants before a long tense stop ( $M=80.24 \mathrm{~ms}$ ), suggesting that there is the opposite directional pattern in duration between $\mathrm{C} 1$ and $\mathrm{C} 2$.

We suggest that this covarying durational pattern occurs between $\mathrm{C} 1$ and $\mathrm{C} 2$ to promote some phonological contrasts in Korean (cf. Diehl et al., 1991). First, lengthening the sonorant before a lax stop may occur to satisfy the robust durational contrast between $C$ vs. $\mathrm{CC}$. Without the lengthening of a sonorant before the lax stop, the $\mathrm{CC}$ sequences involving a lax obstruent may not be substantially different in duration from a single $\mathrm{C}$ or may be substantially different in duration from the other phonologically long consonants like CC.

Furthermore, lengthening the sonorant before a lax consonant may enhance the phonological contrast between a short lax and a long tense stop in this phonological context. To emphasize the short duration of a lax stop at the onset position, Korean takes the strategy of not only making the lax stop short relative to the corresponding tense stop in the same phonological position, but also making the sonorant before the lax stop substantially longer so that the shortness of the lax stop may be enhanced.

Conclusively, this section shows that the timing of segments in Korean is planned and controlled with certain targets. One such target in Korean is the duration of consonant interval between vowels (from offset of the vowel to onset of the following vowel). With a short $\mathrm{C} 2$, the preceding $\mathrm{C} 1$ stretches to achieve the target duration $\mathrm{CC}$ while with a long $\mathrm{C} 2$, no such stretching is necessary. This is a case of phonetic temporal adjustments.

\subsection{Experiment 2}

In this experiment, we will examine the temporal adjustment of the obstruent $\mathrm{C} 2$ when an obstruent occurs at $\mathrm{C} 1$. The relevant context occurs if a morpheme ending with an obstruent $\mathrm{C} 1$ is followed by another morpheme beginning with a lax or a tense obstruent $\mathrm{C} 2$. This sequence will be realized as a [lax stop-tense obstruent] sequence due to Post-Stop Tensing Rule.

\subsubsection{Analysis}

To examine whether closure duration of the consonant sequences plays a role in Post-stop Tensing Rule, we have first measured the duration of [lax stop-tense stop] sequences derived from /lax stop-lax stop/ such as /at-ta/, /ap-pa/, and /ak-ka/. The derived stop sequences are represented as " $\mathrm{t} T$ ". We have also measured the duration of the other consonant sequences like [nasal-lax stop] ("Nt") such as /an-ta/, /am-pa/, and /ay-ka/, and [nasal-tense stop] ("NT*") such as /an-T*a/, /am-P*a/, and /an-K*a/. In order to avoid the possible complication due to the different places of articulation of the segments involved, this section has only considered the consonant sequences that have the same place of articulation. We have also estimated the duration of the [lax stop-lax stop] sequence ("tt") that does not occur in Korean. The duration of " $t \mathrm{t}$ " was estimated by deducting the duration of a tense onset stop from the duration of each " $\mathrm{t} \mathrm{T}^{*}$ " and by adding the duration of a lax onset stop to it. For the duration of a tense onset stop and a lax onset stop in this measurement, the tense onset stop and the lax onset stop in the sequences of "NT*" and "Nt" were used. For each stimulus, there were forty-two tokens ( 7 speakers $\times 1$ type of $\mathrm{Cl}$ (obstruent or nasal) $\times 1$ type of $\mathrm{C} 2$ (lax or tense) $\times 3$ places of articulation of $\mathrm{C} 2$ (bilabial, coronal, velar) $\times 2$ repetitions) $=42$ tokens).

\subsubsection{Results}

Table 2 and Figure 3 presents the durational values of " $\mathrm{tT}$ *" sequence 2 as well as "Nt", "NT*", and "tt" sequences.

Table 2. Mean durations and standard deviations of consonants sequence

\begin{tabular}{c|r|r|r|r}
\hline & \multicolumn{1}{|c|}{$\mathrm{tt}$} & \multicolumn{1}{c|}{$\mathrm{tT}^{*}$} & \multicolumn{1}{c|}{$\mathrm{Nt}$} & \multicolumn{1}{c|}{$\mathrm{NT}^{*}$} \\
\hline Mean $(\mathrm{ms})$ & 109.98 & 166.79 & 149.43 & 169.93 \\
\hline$S D$ & 38.26 & 41.25 & 32.65 & 34.36 \\
\hline
\end{tabular}

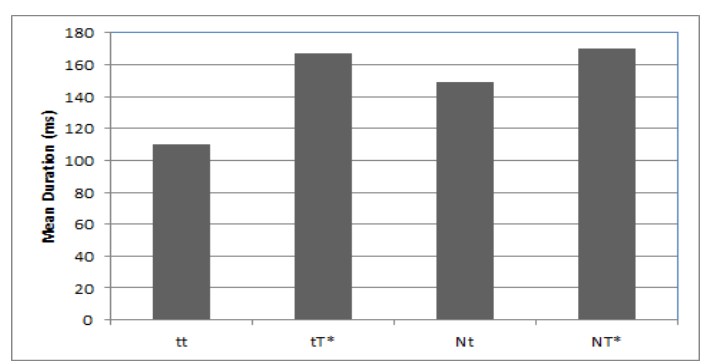

Figure 3. Mean durations (ms) of "tt," $\mathrm{tT}$ *,"Nt, and"NT*"

The durational values of the consonant sequences were submitted to repeated-measures ANOVA. The results show that there is a significant difference in the duration of consonant sequences, $[F(1.58,74.53)=55.73, p<.001$. Degrees of freedom were corrected using Greenhouse-Geisser estimates of sphericity.] Pairwisecomparisons show that the duration of " $t \mathrm{t}$ " is significantly different from those of the other consonant sequences $(p<.001$ for all comparisons). The duration of " $\mathrm{Nt}$ " is also significantly different from those of other consonant sequences ( $p<.001$ from " $\mathrm{tt}$ ", $p<.002$ from "(t) $\mathrm{T}^{*},, p<.001$ from "NT*"). The durations of " $\mathrm{NT}$ *" and 
" $\mathrm{t} T$ *" are not significantly different from each other $(p>.05)$. In short, the duration of " $\mathrm{tT}$ " " is not significantly different from one of the $\mathrm{CC}$ clusters, namely "NT*" while the duration of " $\mathrm{tt}$ " is significantly shorter than the durational values of the consonant sequences like "Nt" or "NT*".

\subsubsection{Discussion}

The statistical results in this section show that the durational values of [lax stop-tense stop] (" $\mathrm{t} \mathrm{T}^{*}$ ") sequences, the forms derived from the underlying /lax stop-lax stop/ sequences by Post-Stop Tensing Rule, are within the durational range of the other consonant sequences such as "NT*" or "Nt". This fact argues that a [lax stop-tense stop] sequence satisfies the distinctive length requirement of CC in Korean. In contrast, the [lax stop-lax stop] ("tt") sequence, the form which would have resulted in if the Post-Stop Tensing Rule did not apply, is significantly shorter than all the other consonant sequences 3 like "Nt" or "NT*".

Based on this observation, we argue that the lengthening of a lax obstruent $\mathrm{C} 2$ occurs after the short lax $\mathrm{C} 1$ in order to achieve the robust contrastive length requirement of the $\mathrm{CC}$ in the surface form. Without the lengthening of an obstruent $\mathrm{C} 2$, the resulting from " $\mathrm{tt}$ " would have failed to meet the durational requirement of CC.

This lengthening of $\mathrm{C} 2$ is referred to as the Post-Stop Tensing Rule $^{4}$ in Korean. The Post-Stop Tensing Rule has long puzzled Korean linguists since there seems to be no phonetic motivation for its application. This paper argues that a lax obstruent at $\mathrm{C} 2$ undergoes the temporal adjustment and becomes a tense obstruent after a short lax stop at $\mathrm{C} 1$ in order to ensure the contrastive long duration of $\mathrm{CC}$ between vowels. Since the category of $\mathrm{C} 2$ is changed from lax to tense, this is a phonological temporal adjustment.

\subsection{Experiment 3: Vowel shortening}

This section will examine another case of temporal adjustments in Korean, namely the vowel shortening before a consonant sequence or before a tense consonant.

\subsubsection{Duration of vowel}

In order to re-examine the vowel shortening before a consonant sequence or a tense obstruent, we measured the vowel duration in various stimuli.

\subsubsection{Analysis}

The durational values of the vowels in "Vt" "VTT*", "VNt", "VNT*", "VLt", and "VLT*"5 were submitted to a repeated measures of ANOVA. For each stimulus, there were forty-two tokens ( 7 speakers $\times 3$ places of articulation of $\mathrm{C} 1$ or $\mathrm{C} 2$ (bilabial, coronal, velar) $\times 2$ repetitions $=42$ tokens) .

\subsubsection{Results}

Table 3 and Figure 4 show the measurements of the vowels in the stimuli.

Table 3. Means (ms) and standard deviations of V

\begin{tabular}{c|c|c|c|c|c|c}
\hline & $\underline{\mathrm{V}}(\mathrm{t})$ & $\underline{\mathrm{V}}\left(\mathrm{T}^{*}\right)$ & $\underline{\mathrm{V}}(\mathrm{Nt})$ & $\underline{\mathrm{V}}\left(\mathrm{NT}^{*}\right)$ & $\underline{\mathrm{V}}(\mathrm{Lt})$ & $\underline{\mathrm{V}}\left(\mathrm{LT}^{*}\right)$ \\
\hline $\begin{array}{c}\text { Mean } \\
(\mathrm{ms})\end{array}$ & 114.62 & 71.76 & 74.00 & 70.24 & 74.60 & 68.29 \\
\hline$S D$ & 35.28 & 25.44 & 21.59 & 22.12 & 21.61 & 21.91 \\
\hline
\end{tabular}

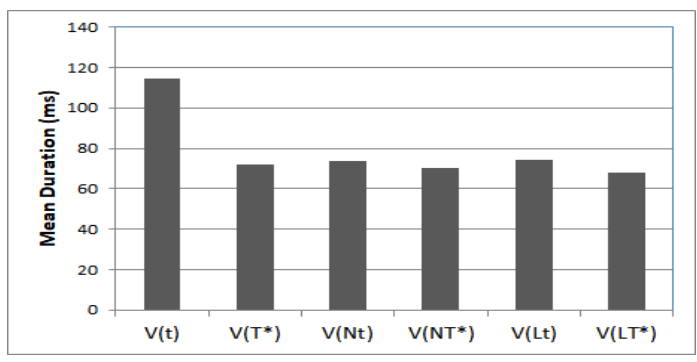

Figure 4. Mean duration (ms) of vowel

One-way Repeated Measures ANOVA shows that there is a significant difference in vowel duration due to the phonological contexts, $[F(3.43,140.77)=55.73, p<.001$. Degrees of freedom were corrected using Greenhouse-Geisser estimates of sphericity.] Pairwisecomparisons show that $\mathrm{V}$ in " $\mathrm{Vt}$ " is significantly longer than the Vs in all the other contexts ( $p<.001$ for all comparisons). There is no other significant difference between the vowels in the other pairwise-comparisons ( $p>05$ for all comparisons).

\subsubsection{Discussion}

The results in this experiment can be summarized as follows: First, the vowel before a single lax consonant "Vt" is significantly longer than the vowels in any other context. Second, the vowel before a single intervocalic tense stop "VT*" is significantly shorter than the vowel before a single lax stop, but does not show significant difference in duration from the vowels before a consonant sequence. This finding confirms the previous finding that an intervocalic tense stop behaves like a geminate or a consonant sequence in vowel shortening (cf. Oh \& Johnson, 1997; Choi \& Jun, 1998, etc.). Third, the duration of the vowel before a consonant sequence does not show a significant difference depending on whether the consonant sequence contains a lax stop as in "VNt" and "VLt" or the tense stop as in "VNT*" and "VLLT*".

\subsubsection{Duration of syllable}

As the vowel becomes short before a consonant sequence or a tense consonant, Choi \& Jun (1998) argued that the vowel

3 In this study, we did not measure the duration of all the CC clusters in Korean. We acknowledge that by using the phrase such as "the duration of the consonant cluster" in this paper we may give the readers the wrong impression that we have measured all the CC clusters in Korean, as one anonymous reviewer pointed out. Though we did not measure the duration of all the consonant sequences such as NN or LL clusters, Choi \& Jun (1998) have measured the duration of NN sequences and showed that NN sequences are more than twice longer than a single nasal consonant in the intervocalic position. As for the duration of LL sequences, we would like to point out that an intervocalic single L is a tap sound, the shortest consonant in Korean.

4 Not only the lax stop C2, but a lax fricative /s/ at C2 also undergoes the Post-Stop Tensing Rule as in /tap-sa/ $\rightarrow$ [tapS*a] 'field trip', though this paper does not deal with the fricatives.

$\mathbf{5}$ In this paper, the underlined segment(s) is the one whose duration is measured for the analysis. 
shortening is motivated by the Closed-Syllable Vowel Shortening and that a single intervocalic tense consonant is a geminate. This section will consider the suggestion by Choi \& Jun (1998) that the vowel shortening in Korean is motivated by Closed-Syllable Vowel Shortening.

\subsubsection{Analysis}

For this purpose, we measured the duration of the syllables before the consonants. Specifically, we measured the duration of the syllables in the stimuli of "V\$t", "V\$T*", "VN\$t", "VN\$T*", "VL\$t", and "VL\$T*". Note that for the measurements of syllables, the intervocalic tense stop $\mathrm{T}^{*}$ in a "VT*V" stimulus is treated as an onset of the second syllable since it is impossible to divide the long closure duration of a tense stop $\mathrm{T}^{*}$ as the coda and the onset. The durations of syllables were submitted to a repeated-measures ANOVA. For each stimulus, there were forty-two tokens (7 speakers $\times 3$ places of the articulation $\times 2$ repetitions $=42$ tokens).

\subsubsection{Results}

Table 4 and Figure 5 show the measurements

Table 4. Means (ms) and standard deviations of syllables

\begin{tabular}{|c|c|c|c|c|c|c|}
\hline & $\underline{V} \$ t$ & VST ${ }^{*}$ & VNSt & VN\$T ${ }^{*}$ & VLSt & VL\$T ${ }^{*}$ \\
\hline $\begin{array}{c}\text { Mean } \\
(\mathrm{ms})\end{array}$ & 114.62 & 71.76 & 195.05 & 154.98 & 178.74 & 144.02 \\
\hline$S D$ & 35.28 & 25.44 & 42.24 & 35.50 & 35.03 & 39.31 \\
\hline
\end{tabular}

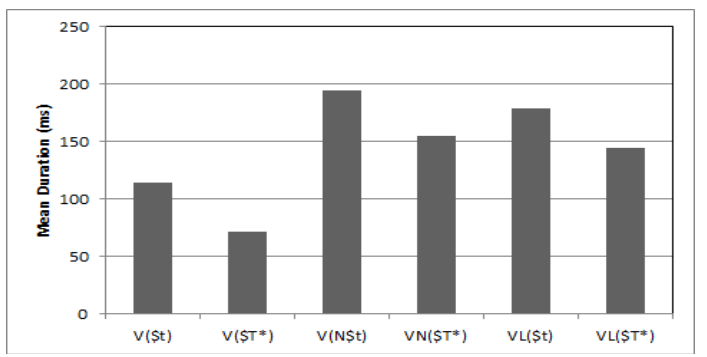

Figure 5. Mean duration(ms) of a syllable

With the dependent variable of syllable duration, one-way repeated measures ANOVA shows that there is a significant difference in the syllable length $[F(5,205=225.80, p<.001)]$.

Pairwise-comparisons show that the syllable duration of "V\$t" and " $\underline{\mathrm{V}} \mathrm{T}$ "" are significantly different from all the other syllable durations $(p<.001$ for all comparisons) and from each other $(p<.001)$. Syllable durations of "VN\$T", and "VL\$T", are also significantly different from all the other syllable durations $(p<.001$ for all comparisons) but they are not significantly different from each other $(p>.05)$. Syllable durations of "VN\$t" and "VL\$t" are also significantly different from all the other syllable durations $(p<.001$ for all comparisons) and they are significantly different from each other as well $(p<.004)$. In short, the results show that syllable durations are progressively longer as in "V $\mathrm{V} \mathrm{T}^{*}$ " $<$ "V $\$ \mathrm{t}$ " $<$

\section{"VN\$T", "VL\$T"”<"VL\$t"<"VN\$t"}

In short, syllables with a coda are significantly longer than syllables without a coda like "V $\$ \mathrm{t}$ " or "V $\$ \mathrm{~T}$ ". with a coda, the syllables before a lax stop is significantly longer than the syllables before a tense stop. In addition, the syllable duration before the tense stop " $\mathrm{VT}$ ", is significantly shorter than that before the lax stop " $\underline{\mathrm{V}} \mathrm{t}$ ".

\subsubsection{Discussion}

Choi \& Jun (1998) have argued that vowel shortening occurs before a consonant sequence and that this is a case of ClosedSyllable Vowel Shortening. They have also argued that an intervocalic tense consonant is a geminate and that the short vowel before a tense consonant is a case of Closed-Syllable Vowel Shortening.

The suggestion by Choi \& Jun (1998), however, brings up some issues. Closed-Syllable Vowel Shortening implies that the vowel is shortened in a closed syllable due to the temporal adjustment for the presence of the coda at the syllable level. In other words, the vowel is shortened before a coda so that a certain near-equal durational value of the syllable could be maintained. If so, one may further expect that the durational values of the vowel and the following coda may also show an inverse relationship with each other: The vowel should be shorter before a phonetically long coda than before a phonetically short coda.

Korean presents a testing case. Coda sonorants in Korean show a significant difference in length depending on the context: The sonorant before the lax stop is significantly longer than it is before the tense stop. Therefore, we expect that this systematic length difference among coda sonorants is reflected on the vowel length such that the extra-short vowel occurs before the phonetically long sonorant coda.

However, this is not the case. We have shown that vowel does not become extra-short before the lengthened coda in Section 2.4.1. The fact that vowel did not become extra-short before the lengthened coda is reflected in the duration of the syllables. The durational values of the syllable before a lax stop, VN\$t (195.05 ms) and VL\$t (178.74 ms), are significantly longer than those before the tense stop, VN\$T* (154.98 ms) and VL\$T* (144.02 ms). The vowel shortening cannot be well explained with Closed-Syllable Vowel Shortening.

Second, it is questionable whether the short vowel before a tense stop is a case of Closed-Syllable Vowel Shortening. For the short vowel before a tense stop to be a case of Closed-Syllable Vowel Shortening, the intervocalic tense stop should be a geminate, one member of which is syllabified as a coda of the first syllable. The authors, who are native speakers of Korean, agree that the intervocalic tense stop is a phonologically long consonant, but do not think that the intervocalic tense stop consists of a coda and an onset like a regular consonant sequence or a geminate 6 . In fact, the authors wonder whether an intervocalic tense consonant contains a coda at all.

Without the coda, the preceding vowel cannot be a closed

6 Some phonologists (Kim, 1970; Kim-Renaud, 1974; Sohn, 1987; Cho, 1990) assume that a tense consonant is a single, not a geminate segment even in the intervocalic position. If a tense consonant is a single segment as they assume, it cannot trigger Closed-Syllable Vowel Shortening either since it is syllabified as an onset, not as a coda in the intervocalic position. 
syllable, and thus there can be no trading relationship between the shortened vowel and the presence of the coda in the case of the intervocalic consonant.

\subsubsection{A unit $\{\operatorname{VC1}(\mathrm{C} 2)\}$}

Since the timing unit "syllable" may not be a proper unit in accounting for the vowel shortening in Korean, this section examines whether a different entity like the duration from the onset of the vowel to the onset of the following vowel (hereafter, $\{\mathrm{VC} 1(\mathrm{C} 2)\})$ can be a better candidate for the vowel shortening.

\subsubsection{Analysis}

For this purpose, the durations of $\{\mathrm{VC} 1(\mathrm{C} 2)\}$ of six stimuli, Vt, $\mathrm{VT}^{*}, \mathrm{VNt}, \mathrm{VNT}^{*}, \mathrm{VLt}$, and $\mathrm{VLT}^{*}$, were measured and submitted to a repeated-measures ANOVA. For each stimulus, the tokens were forty- two tokens ( 7 speakers $\times 3$ places of the articulation $\times 2$ repetitions $=42$ tokens).

\subsubsection{Results}

With the dependent values of $\{\mathrm{VCl}(\mathrm{C} 2)\}$ duration, one-way Repeated Measures ANOVA shows that there is a significant difference among stimuli, $[F(5,205=86.58, p<.001)$. Table 5 and Figure 6 show the measurements.

Pairwise-comparisons show that Vt is significantly different from all the other stimuli $\left(p<.001\right.$ for all comparisons). $\mathrm{VT}^{*} 7$ does not show a significant difference from VNt and VLt $(p>.05)$ but it shows a significant difference from $\mathrm{VNT}^{*}$ and $\mathrm{VLT}^{*}(p<.001$ for all). VLt does not show a significant difference from $\mathrm{VNT}^{*}(p>.05)$ but it shows a significant difference from $\mathrm{VLT}^{*}(p<.001)$. VNT shows a significant difference from $\mathrm{VLT}^{*}(p<.002)$.

In short, the results show that $\{\mathrm{VCl}(\mathrm{C} 2)\} \mathrm{s}$ are progressively longer as in $\mathrm{Vt}<\mathrm{VT}^{*}$, VNt, $\mathrm{VLt}<\mathrm{VNT}^{*}(=\mathrm{VLt})<\mathrm{VLT}^{*}$. The unit $\{\mathrm{VC} 1(\mathrm{C} 2)\}$ shows that the intervocalic tense stop behaves like a consonant sequence for the vowel shortening.

Table 5. Means (ms) and standard deviations of $\{\mathrm{VC} 1(\mathrm{C} 2)\}$

\begin{tabular}{c|c|c|c|c|c|c}
\hline & $\mathrm{Vt}$ & $\mathrm{VT}^{*}$ & $\mathrm{VNt}$ & $\mathrm{VNT}^{*}$ & $\mathrm{VLt}$ & $\mathrm{VLT}^{*}$ \\
\hline $\begin{array}{c}\text { Mean } \\
(\mathrm{ms})\end{array}$ & 170.81 & 215.12 & 223.43 & 240.17 & 227.55 & 256.86 \\
\hline$S D$ & 34.56 & 35.93 & 41.62 & 40.70 & 36.01 & 44.34 \\
\hline
\end{tabular}

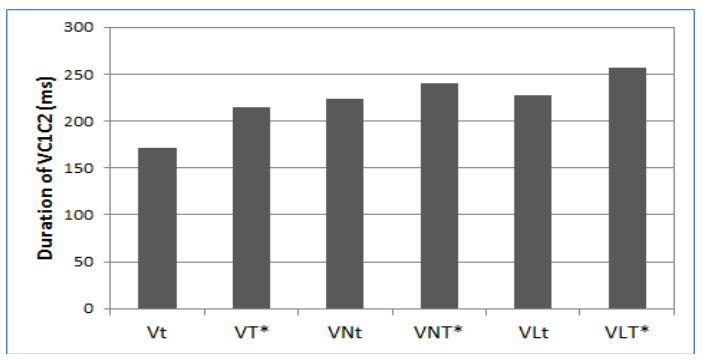

Figure 6. Mean duration(ms) of $\mathrm{VC1}(\mathrm{C} 2)$

\subsubsection{Discussion}

Unlike the "syllable", the entity $\{\mathrm{VC}(\mathrm{C} 2)\}$ is not a prosodic unit in phonology. It does not require the presence of a coda but rather the presence of a following onset consonant. Let us examine whether this unit better explains the vowel shortening in Korean than Closed-Syllable Vowel Shortening does.

In Section 2.4.2, we have suggested that first, Closed-Syllable Vowel Shortening cannot explain the vowel shortening before a tense consonant because the long tense consonant does not have a coda. In contrast, this unit $\{\mathrm{VC} 1(\mathrm{C} 2)\}$ can explain the vowel shortening before a tense consonant very easily: The vowel is shortened before a phonologically long consonant within the unit and a phonologically long consonant includes a consonant sequence, a geminate or a long tense obstruent.

Second, Closed-Syllable Vowel Shortening argues that the vowel becomes short in a closed syllable in order to accomplish a certain near-equal interval at the syllable level. If so, it further predicts that a relatively long vowel occurs before a phonetically short coda in a syllable while a relatively short vowel occurs before a phonetically long coda in a syllable just like in English. However, we have shown that this is not the case in Korean: Vowels do not show a significant difference in length regardless of whether they are followed by a phonetically long coda such as $/ \mathrm{n} /$ in /anta/ or by a phonetically short coda such as $/ \mathrm{n} /$ in $/ \mathrm{anT}^{*} \mathrm{a} /$. This is a problem for the Closed-Syllable Vowel Shortening.

In contrast, the unit $\{\mathrm{VC} 1(\mathrm{C} 2)\}$ presents no such problem. Note that the consonant(s) in the unit $\{\mathrm{VC} 1(\mathrm{C} 2)\}$ have only two distinctive length difference, namely a short $\mathrm{C}$ vs. a long $\mathrm{CC}$ consonant. All the long consonants such as Nt, Lt, NT*, LT*, or T* are shown in this paper to have more or less near-equal length. As such, the vowel in this unit is expected to have only distinctive length difference: A long vowel before a single lax consonant and a short vowel before a long consonant such as $\mathrm{Nt}, \mathrm{Lt}, \mathrm{NT}^{*}, \mathrm{LT}^{*}$, or $\mathrm{T}^{*}$. This is in fact what is observed in Korean. Therefore, this unit $\{\mathrm{VC} 1(\mathrm{C} 2)\}$ better explains the vowel shortening in Korean than Closed-Syllable Vowel Shortening does.

The fact that $\{\mathrm{VC1}(\mathrm{C} 2)\}$ shows a better near-equal length among various stimuli than the unit "syllable" can be confirmed by the measurements of the stimuli as well. Consider Table 6.

Table 6. Percentages of Syll/V(t) and $\{\mathrm{VC} 1(\mathrm{C} 2)\} / \mathrm{Vt}$

\begin{tabular}{|c|c|c|c|c|}
\hline & \multicolumn{2}{|c|}{ Syll } & \multicolumn{2}{|c|}{$\mathrm{VC} 1(\mathrm{C} 2)$} \\
\hline & $\begin{array}{c}\text { Mean } \\
(\mathrm{ms})\end{array}$ & $\begin{array}{c}\text { Percent } \\
(\%)\end{array}$ & Mean (ms) & $\begin{array}{c}\text { Percent } \\
(\%)\end{array}$ \\
\hline $\mathrm{Vt}$ & 114.62 & 100 & 170.81 & 100 \\
\hline VT* & 71.76 & 63 & 215.12 & 126 \\
\hline $\mathrm{VNt}$ & 195.05 & 170 & 223.43 & 131 \\
\hline $\mathrm{VNT}^{*}$ & 154.98 & 135 & 240.17 & 141 \\
\hline VLt & 178.74 & 156 & 227.55 & 133 \\
\hline VLT* & 144.02 & 126 & 256.86 & 150 \\
\hline
\end{tabular}

In Table 6, the percentage of syllable length of each stimulus compared to the syllable length of the stimulus "V(t)" shows the

7 In this study, we did not directly compare the duration of $/ \mathrm{t}-\mathrm{t} / \mathrm{in} / \mathrm{at}-\mathrm{ta} /\left(\right.$ realized as $\left[\mathrm{a}(\mathrm{t}) \mathrm{T}^{*} \mathrm{a}\right]$ ) with the duration of $/ \mathrm{T}^{*} /$ in $/ \mathrm{aT}^{*} \mathrm{a} /$ (realized as [aT*a]). However, we have shown that the average duration of $/ \mathrm{t}-\mathrm{t} /$ in $/ \mathrm{at}-\mathrm{ta} /$ is $166.79 \mathrm{~ms}$ in Experiment 1 while the average duration of $/ \mathrm{T}^{*} / \mathrm{in} / \mathrm{aT} * \mathrm{a} /$ is $144.36 \mathrm{~ms}$ in Experiment 3. The speakers in this study produced $/ \mathrm{t}-\mathrm{t} /$ in $/ \mathrm{at}-\mathrm{ta} /$ longer than $/ \mathrm{T}^{*} /$ in $/ \mathrm{a} \mathrm{T}^{*} \mathrm{a} /$, possibly due to the orthography. 
range of $63 \%$ to $170 \%$. On the other hand, the percentage of $\{\mathrm{VC} 1(\mathrm{C} 2)\}$ length of each stimulus compared to the $\{\mathrm{VCl}(\mathrm{C} 2)\}$ length of stimulus "Vt" shows the range of $126 \%$ to $150 \%$. The unit $\{\mathrm{VCl}(\mathrm{C} 2)\}$ shows better near-equal length among various stimuli than the unit "syllable" does.

Conclusively, we argue that Korean speech be planned and controlled. Vowel shortening occurs in order to make the unit $\{\mathrm{VCl}(\mathrm{C} 2)\}$ of the stimuli be near-equal lengths.

\section{Conclusion}

This paper has shown that Korean lax and tense stops influence the duration of the nearby segments: The short lax stop makes the nearby segments stretch while a long tense stop makes the nearby segments compressed. Specifically, this paper has shown that the short lax stop $\mathrm{C} 2$, but not a long tense stop $\mathrm{C} 2$, makes the preceding sonorant $\mathrm{C} 1$ significantly long, that a short lax stop $\mathrm{C} 1$ makes the following lax consonant $\mathrm{C} 2$ significantly long, making it a categorically different long tense consonant, and that the vowel is significantly shortened before a phonologically long consonant like an intervocalic tense stop or a consonant sequence, but not before a short lax stop.

Based on these findings, this paper argues that temporally co-varying segments occur in Korean at the function of a lax or tense stop in order to accomplish the requirements of two intervals. One such interval is the interval from the offset of the vowel to the onset of the vowel, namely, the duration of the consonants between vowels. In order to robustly preserve the phonological length difference between a short (C) and a long consonant (CC), Korean employs strategies of segment lengthening. That is, if the distinctive long duration of $\mathrm{CC}$ can be violated due to the inherent short duration of a lax segment in the sequence, the other consonant in the sequence lengthens to compensate for this temporal loss in the sequence. We have shown a case of phonetic temporal adjustment (Experiment 1) in which a sonorant such as a nasal or a lateral stretches before a short lax stop, and a case of phonological temporal adjustment (Experiment 2) in which the short lax obstruent at $\mathrm{C} 2$ stretches and becomes a long tense stop after a short lax stop at $\mathrm{C} 1$.

The other interval that influences the segment duration in Korean is the interval from the onset of the vowel to the onset of the following vowel, namely $\{\mathrm{VC} 1(\mathrm{C} 2)\}$. In order to make this interval of near-equal length, some trading relationship was observed between a vowel and the following consonants: When a phonologically long consonant follows a vowel, the vowel becomes short. If not, no such compression of the vowel is observed.

In summary, the present investigation attempted to demonstrate that the timing of speech in Korean is planned and controlled with specific targets, i.e., the duration of consonant interval between vowels and the interval from the onset of the vowel to the onset of the following vowel. These two targets are not phonological units like "syllables" or "stresses", but rather contain the onset that does not bear weight in phonology.

Steriade (1999) argues that consonantal phonotactics are better explained with string-based conditions like the positional differences than with phonological units like a syllable. This paper also showed that string-based conditions such as whether a segment occurs before a lax stop or after a lax stop or whether a vowel occurs before a single consonant or before a consonant sequence better explain the different length of the segments of interest than the phonological units like "syllables" do. The entities we suggested, the consonant interval between vowels and the vowel-to-vowel interval $\{\mathrm{VC} 1(\mathrm{C} 2)\}$, are well perceived as they are demarcated by the highly perceptual segment, a vowel.

Previous research including Ramus et al. (1999) and Low et al. (2000), has also argued for the entities that involve the vowel. We suggest that these entities are determined not only by universal constraints but also by language-specific constraints.

Further investigation is necessary to find out whether the same lengthening and shortening occurs when a lax and a tense fricative are involved, though we believe that they also show the same pattern as the stops in the durational variation.

\section{References}

Abercrombie, D. (1967). Elements of general phonetics. Chicago, IL: Aldine.

Bolinger, D. (1965). Forms of English: Accent, morpheme, order. Cambridge, Ma: Harvard University Press.

Cho, Y. (1990). The parameters of consonantal assimilation (Doctoral dissertation). Stanford University, Stanford, CA.

Choi, S., \& Jun, J. (1998). Are Korean fortis and aspirated consonants geminates? Linguistic Research, 34(3), 521-546.

Delattre, P. (1966). A comparison of syllable length conditioning among languages. International Review of Applied Linguistics in Language Teaching, 4(3), 183-198.

Diehl, R., Kluender, K., Walsh, M., \& Parker, E. (1991). Auditory enhancement in speech perception and phonology. In R. Hoffman \& D. Palermo (eds.), Cognition and the symbolic processes: Applied and ecological perspectives (pp. 59-76). Hillsdale, JM: Lawrence Erlbaum Associates.

Ham, W. (2001). Phonetic and phonological aspects of geminate timing. New York, NY: Routledge.

Han, J. (1996). The Phonetics and phonology of "tense" and "plain" consonants in Korean (Doctoral dissertation). Cornell University, Ithaca, NY.

Han, M. (1992). The timing control of geminate and single stop consonants in Japanese: A challenge for nonnative speakers. Phonetica, 49(2), 102-127.

Idemaru, K., \& Guion, S. (2008). Acoustic covariants of length contrast in Japanese stops. Journal of the International Phonetic Association, 38(2), 167-186.

Jun, S. (1993). The phonetics and phonology of Korean prosody (Doctoral dissertation). Ohio State University, Columbus, $\mathrm{OH}$.

Jun, S. (1995). Asymmetrical prosodic effects on the laryngeal gesture in Korean. In B. Connell (Ed.), Papers in laboratory phonology 4 (pp. 235-253). Cambridge, UK: Cambridge University Press.

Kim, C. (1970). A theory of aspiration. Phonetica, 21(2), 107-116.

Kim-Renaud, Y. (1974). Korean consonantal phonology (Doctoral dissertation). University of Hawaii, Honolulu, HI.

Kim, M. (2000). Segmental and tonal interactions in English and Korean: A phonetic and phonological study (Doctoral dissertation). The University of Michigan, Ann Arbor, MI.

Kim, T. (2014). A phonetic study on two successive stop consonants with the same place in Korean. Journal of Language Sciences, 21(3), 45-64.

Lisker, L. (1957). Closure duration and the intervocalic voiced voiceless distinction in English. Language, 33(1), $42-49$. 
Low, L., Grabe, E., \& Nolan, F. (2000). Quantitative characterizations of speech rhythm: Syllable-timing in Singapore English. Language and Speech, 43(4), 377-401.

Maddieson, I. (1985). Phonetic cues to syllabification. In V. Fromkin (ed.), Phonetic linguistics: Essays in honor of Peter Ladefoged (pp. 203-221). Orlando, FL: Academic Press.

Oh, J. (2015). A study on isochronism in the rhythm of the Korean language. Korean Linguistics, 68, 95-115.

Oh, M., \& Johnson, K. (1997). A phonetic study of Korean intervocalic laryngeal consonants. Speech Sciences, 1(1), 83-101.

Pike, K. (1945). The Intonation of American English. Ann Arbor, MI: University of Michigan Press.

Ramus, F., Nespor, M., \& Mehler, J. (1999). Correlates of linguistic rhythm in the speech signal. Cognition, 73(3), 265-292.

Sohn, H. (1987). Underspecification in Korean phonology (Doctoral dissertation). University of Illinois, Champaign, IL, Urbana.

Steriade, D. (1999). Alternatives to syllable-based accounts of consonantal phonotactics. Proceedings of LP' 98 (pp. 205-245). Columbus, $\mathrm{OH}$.

Silva, D. (1992). The phonetics and phonology of stop lenition in Korean (Doctoral dissertation). Cornell University, Ithaca, NY.

\section{- Hyunsook Kang}

Professor, Dept. of English Language \& Culture, Hanyang

University

55 Hanyangdaehak-ro, Sangnok-gu, Ansan 15588, Korea

Tel: +82-31-400-5348

Email: hskang@hanyang.ac.kr

Fields of interest: Phonology, Phonetics, L2 Acquisition

- Tae-kyung Kim, Corresponding author

Associate Professor, Center for Integrated General Education,

Hanyang University

55 Hanyangdaehak-ro, Sangnok-gu, Ansan 15588, Korea

Tel: +82-31-400-5776

Email: kimtk@hanyang.ac.kr

Fields of interest: Phonology, Phonetics, L2 acquisition

\section{Appendix}
1. /ataimnita/
[adaimnida]
2. /aT*aimnita/
[aT*aimnida]
3. /apaimnita/
[abaimnida]
4. /aP*aimnita/
[aP*aimnida]
5. /akaimnita/
[agaimnida]
6. /aK*aimnita/
[aK*aimnida]
7. /anaimnita/
[anaimnida]
8. /annaimnita/
[annaimnida]
9. /amaimnita/
[amaimnida]
10. /ammaimnita/
11. /alaimnita/
[ammaimnida]
12. /allaimnita/
[araimnida]
13. /at-taimnita/
[allaimnida]
14. /ap-paimnita/
[aT*aimnida]
15. /ak-kaimnita/
16. /antaimnita/
17. /anT*aimnita/
[aP*aimnida]
[aK*aimnida]
[andaimnida]
18. /ampaimnita/
19. /amP*aimnita/
20. /ankaimnita/
21. /ayK*aimnita/
22. /altaimnita/
23. /alT*aimnita/
24. /alpaimnita/
25. /alP*aimnita/
26. /alkaimnita/
27. /alK*aimnita/
[anT*aimnida]
[ambaimnida]
[amP*aimnida]
[angaimnida]
[anK*aimnida]
[aldaimnida]
[alT*aimnida]
[albaimnida]
[alP*aimnida]
[algaimnida]
[alK*aimnida] 